\title{
Portfólio reflexivo: ferramenta inovadora de avaliação formativa na educação em saúde
}

\author{
Reflective portfolio: innovative tool for training evaluation in health education \\ Portfólio reflexivo: herramienta innovadora de evaluación formación en la educación en \\ salud
}

Fernanda de Nazaré Cardoso dos Santos Cordeiro ${ }^{1 *}$, José Antonio Cordero da Silva1.

\section{RESUMO}

Objetivo: Realizar uma revisão narrativa sobre o portfólio reflexivo como ferramenta avaliativa no processo de ensino-aprendizagem dos cursos de formação de profissionais de saúde. Revisão Bibliográfica: $O$ portfólio é uma ferramenta inovadora que consiste na coleção dos melhores trabalhos do estudante e que revela seu progresso e as realizações. Mostra também os pontos fracos e que necessitam de melhorias na trajetória do aluno, mediado pelo professor com a finalidade de formar profissionais críticos e reflexivos que, a partir das experiências vivenciadas, possam tomar decisões e criar soluções para os problemas na prática, articulando com a teorização. O portfólio facilita a avaliação criando um ambiente favorável a discussão, avaliação interpares e autoavaliação através de feedback constante. Considerações finais: Conclui-se que o portfólio é ferramenta útil e potencializadora de aprendizagem significativa levando ao desenvolvimento pessoal e profissional.

Palavras-Chave: Aprendizagem, Avaliação educacional, Diário.

\begin{abstract}
Objective: To carry out a narrative review on the reflexive portfolio as an evaluation tool in the teachinglearning process of the training courses for health professionals. Bibliographic Review: The portfolio is an innovative tool that consists of the collection of the best student work and revealing its progress and achievements. It also shows the weaknesses that need improvement in the trajectory of the student, mediated by the teacher in order to train critical and reflexive professionals who, based on their experiences, can make decisions and create solutions to problems in practice, articulating with theorizing. The portfolio facilitates assessment by creating a supportive environment for discussion, peer review and self-assessment through constant feedback. Final considerations: It is concluded that the portfolio is a useful and potentiating tool for meaningful learning leading to personal and professional development.
\end{abstract}

Key words: Learning, educational assessment, Diary.

\section{RESUMEN}

Objetivo: Realizar una revisión narrativa del portafolio reflexivo como herramienta de evaluación en el proceso de enseñanza-aprendizaje de los cursos de capacitación para profesionales de la salud. Revisión bibliográfica: El portafolio es una herramienta innovadora que consiste en la recopilación del mejor trabajo estudiantil y la revelación de sus avances y logros. También muestra las debilidades que necesitan mejorar en la trayectoria del estudiante, mediada por el maestro para capacitar a profesionales críticos y reflexivos que, en base a sus experiencias, pueden tomar decisiones y crear soluciones a problemas en la práctica, articulando con teorizar El portafolio facilita la evaluación al crear un entorno de apoyo para la discusión, la revisión por pares y la autoevaluación a través de la retroalimentación constante. Consideraciones finales: se concluye que la cartera es una herramienta útil y potenciadora para el aprendizaje significativo que conduce al desarrollo personal y profesional.

Palabras clave: Aprendizaje, Evaluación educativa, Diario.

${ }^{1}$ Universidade do Estado do Pará (UEPA), Belém-Pará. *E-mail: fernandancscordeiro@hotmail.com 


\section{INTRODUÇÃO}

A aprendizagem é um processo construído conjuntamente através das experiências de professor e aluno (STABENOW RSC, et al., 2015). As atuais diretrizes nacionais e internacionais de educação apresentam novos modos de pensar e praticar o processo de ensino-aprendizagem a partir das demandas do atual cenário profissional. É uma transformação do modelo atual tecnicista baseado em simples transmissão de conhecimentos, com alunos passivos, receptores e memorizadores de conceitos para uma formação por competências com conhecimentos, habilidades e atitudes em cenários mutantes e abertos. Neste processo deve haver compreensão de conhecimentos adquiridos, elaboração de críticas, comunicação clara e explícita, trabalho em equipe, compromisso ético e aplicação dos conhecimentos na prática (COTTA RMM, et al., 2015).

A formação em saúde deve estar voltada para o cuidado integral e a avaliação deve valorizar o aperfeiçoamento contínuo. O cenário atual brasileiro tem uma cultura avaliativa pobre que privilegia aspectos somativos, porém a avaliação deve ser abrangente e priorizar competências com habilidades cognitivas, psicomotoras e afetivas, deve levar a produção de um impacto educacional, com reflexão sobre a prática, demonstrando as fragilidades e potencialidades, traçando um caminho a ser percorrido (STELET BP, et al., 2016). Através de métodos inovadores voltados para formação e capacitação de profissionais com novas formas de ensino-aprendizagem para desenvolver a capacidade de reflexão sobre problemas reais pode-se formular ações originais e criativas para transformar a realidade (FORTE FDS, et al., 2015).

Os cursos que formam profissionais de saúde devem gerar profissionais com formação generalista e humanista, tendo como ponto forte a visão crítica e reflexiva da realidade, atuando no Sistema Único de Saúde (SUS) visando a atenção a saúde de forma integral. Para isso as metodologias ativas de ensinoaprendizagem centradas no aluno adquirem importante papel com o professor como facilitador na construção do conhecimento e o aluno vivenciando situações reais. Neste contexto deve haver integração entre docente e aluno para alcançar a competência, aplicando o conhecimento e refletindo na ação, com formação de pessoas capazes de evoluir e aprender (SILVA CJV, et al., 2016).

Para atender a essas demandas de aprendizagem significativa configura-se uma ferramenta inovadora que leva o estudante a se tornar sujeito ativo no processo de construção de seu conhecimento, desenvolvendo o pensamento crítico e reflexivo, auxiliando na tomada de decisões levando a soluções dos problemas vivenciados. Esta ferramenta é o portfólio reflexivo, que permite o acompanhamento de forma longitudinal da evolução do discente, demonstrando pontos fortes e fracos, que necessitam de melhorias (TONNI, et al., 2016).

Nesse sentido, e pelo fato de ainda ser algo pouco conhecido nas formações em geral, é necessário aprofundar-se no estudo desta nova ferramenta, seu conceito, finalidades, vantagens e desvantagens para a construção de um profissional de saúde que consiga transformar seu contexto social em benefício da comunidade. Por isso este trabalho tem como objetivo realizar uma revisão narrativa sobre o portfólio reflexivo, demonstrando seu valor e seu uso como ferramenta avaliativa na formação em saúde.

\section{REVISÃO BIBLIOGRÁFICA}

\section{A avaliação e a formação em saúde}

Os modelos educacionais atuais de ensino estão sendo substituídos para estimular a construção do conhecimento pelo estudante tendo o docente como o facilitador para alcançar a aprendizagem significativa, com formação de um profissional capaz de desenvolver múltiplas competências (CESARIO JB, et al., 2016; BERNARDI MC, et al., 2015). A mudança do foco do processo de educação necessita da introdução de metodologias e instrumentos pedagógicos ativos de ensino, aprendizagem e avaliação para dar origem a estudantes com autonomia, criatividade e responsabilidade. Com isso o aluno aprende a buscar soluções e resolver problemas profissionais, adquirindo o protagonismo de seu próprio processo de ensinoaprendizagem. Há também o compartilhamento de saberes e experiências entre professores e estudantes, com enfoque nos princípios e diretrizes do SUS, considerando as necessidades de saúde individuais e coletivas (COTTA, RMM, et al., 2016). 
O aprendizado efetivo ocorre através da reflexão sobre a realidade, identificando os problemas e analisando-os criticamente (FRANK A, 2017). A implementação de métodos ativos e inovadores visando a mudança das práticas tradicionais de avaliação, ensino e aprendizagem e formas diferentes de conceber o modelo educativo, exigem mudanças e, também exigem que a instituição crie condições para capacitar as pessoas para suprir as demandas da sociedade. Essa nova conjuntura demanda cenários de educação com transformações de paradigmas apresentando um modelo educativo baseado na formação por competências, onde quem está no centro das ações educativas é o aluno (COTTA, RMM, et al., 2016).

Aos profissionais de saúde exige-se cada vez mais flexibilidade e capacidade de adaptação, com apropriação crítica do conhecimento (COTTA, RMM, et al., 2016). A intenção é ressignificar a prática avaliativa para superar a perspectiva meramente classificatória, conferindo um sentido mais diagnóstico e formativo, mudando também o instrumental, permitindo melhoras no desempenho acadêmico com educação permanente (FROTA MMA, et al., 2011). Ocorre a formação de profissionais cidadãos, com despertar da criatividade em situações como problemas a resolver se aproximando o máximo possível da vida real. Para isso é necessário também uma renovação profunda da docência, sendo que o ponto central deixa de ser o ensino e passa a ser o processo de aprendizagem com aprendizado autônomo, incentivando a liberdade e a busca de identidade no processo de ensino-aprendizagem (COTTA RMM, et al., 2013; ROSSI NF, et al., 2014).

Aprender é adquirir conhecimentos, habilidades, é um processo ativo. Educar é muito mais que treinar, é um processo de construção e reconstrução de conhecimento, com alunos capazes de atuarem produtivamente e de forma comprometida em seus ambientes sociais e em suas atividades profissionais (BOTTI SHO, et al., 2009). O saber não pode ser considerado como estático. Evolução é a peça-chave do ensino e aprendizagem, professores tem que ter a clareza do que os alunos aprenderam e os alunos têm uma referência do que necessitam aprender (VIEIRA VMO, 2002).

O ato de avaliar é uma atividade intrínseca e indissociável a qualquer tipo de ação que vise proporcionar mudanças. Nesse sentido a avaliação é uma atividade constituinte da ação educativa, seja na avaliação do ensino, seja na avaliação da aprendizagem (BAU C, 2019). A avaliação precisa ser abrangente e dinâmica, priorizando a construção de um perfil de profissionais comprometidos em busca de respostas, estratégias e mecanismos que auxiliem o enfrentamento de problemas reais, alimentado pelas experiências e vivências, a partir da análise crítica e reflexiva. A avaliação do estudante nas profissões de saúde tem papel importante na sua formação, pois avaliar significa obter informações que vão ajudar nas tomadas de decisões e planejamento, reforçando o aprendizado, com desenvolvimento e aprimoramento de competências profissionais. É necessário, portanto, um método inovador, com registro das reflexões e impressões, favorecendo o aprendizado efetivo das experiências (CARDOSO DAS, et al., 2015).

A avaliação é componente integral do currículo e deve ser concebida para contribuir para a aprendizagem - promover autoconsciência e desenvolver habilidades metacognitivas. O estudante deve pensar o que está aprendendo e reinterpretar habilidades e entendimentos conceituais, percebendo a utilidade e se engajando. Há um alinhamento de estratégias de avaliação inovadoras e autênticas para promover o aprendizado estruturado e efetivo para consolidar e aplicar aprendizagem em uma preparação para a transição para vida profissional (SIDEBOTHAM M, et al., 2018).

Com ferramentas avaliativas busca-se habilidades para resolver problemas, raciocínio clínico e crítico, facilitando a prática baseada em evidências e não em rotinas, com benefício na escrita reflexiva, no desenvolvimento de habilidades de pensamento crítico para integrar e consolidar conhecimento e experiência (SIDEBOTHAM M, et al., 2018).

\section{O portfólio}

O portfólio é mais conhecido no campo da arte e da arquitetura demonstrando os melhores trabalhos de um profissional (CLARKE, et al., 2016). Difundiu-se em âmbito universitário desde os anos 1990, utilizado para qualificar avaliações formativas e somativas e contribuindo para o acompanhamento do progresso individual (STELET BP, et al., 2016). Seu uso no ensino da educação tem sido usado substancialmente, 
porém no ensino da saúde é mais recente, com mais de 5 mil citações em programas de graduação incluindo medicina, enfermagem, fisioterapia, fonoaudiologia e odontologia. É uma metodologia diferenciada e diversificada de monitoramento e avaliação do processo ensino-aprendizagem (PADOVANI O, et al., 2015).

O portfólio é uma coleção que mostra os esforços, progressos e realizações do estudante, é uma documentação rica de experiências ao longo do tempo. A sua construção inclui autocorreção e desenvolvimento profissional, respeitando a individualidade e demonstrando seu processo de aprendizagem, num contexto de evolução e superação. Propõe questões estimuladoras e desafiantes e revela a compreensão do estudante favorecendo o seu envolvimento num processo criativo. Tem o olhar do professor e a perspectiva do aluno sobre os caminhos realizados, conquistas e dificuldades. Este instrumento facilita a compreensão dos sentimentos, interesses e maneiras de ver e pensar sobre as conquistas, dando condições de se expressar. $O$ docente instiga o desenvolvimento integral para desenvolver pensamento intuitivo, criativo, lógico e prático, com flexibilidade e capacidade de adaptação as constantes mudanças (COTTA RMM, et al., 2015).

O portfólio é uma fonte de informações sobre aprendizado e desenvolvimento socio-afetivo, das relações, da capacidade de resolução de problemas e conflitos, que permite valorizar as produções para que ocorra avanços e proporciona ao professor a análise de suas ações educativas (STABENOW RSC, et al., 2015). É um método de estímulo ao pensamento reflexivo e potencial processo pedagógico para estudantes se transformarem em pessoas ativas, investigadores críticos e abertos ao diálogo e ao novo (COTTA RMM, et al., 2015).

A organização do portfólio pode variar de acordo com a instituição que o utiliza, mas sempre com o objetivo de acompanhar a aprendizagem e o desenvolvimento, servindo também como avaliação formativa, favorecendo o acompanhamento longitudinal do processo de aprendizagem vivenciado. O portfólio demonstra evidências do processo, como participação, reflexão, necessidades e conquistas. Além de ser ferramenta que respeita a individualidade, já que é um processo de escolha das atividades armazenadas consideradas as melhores produções (STABENOW RSC, et al., 2015).

O estudante busca, seleciona, pensa, analisa, reflete, estabelece relações, julga, faz descobertas e gera novas ideias, efetivando o pensamento compreensivo, crítico e criativo e tomada de consciência de suas capacidades e conhecimentos, permite o compartilhamento das ideias, e também permite que revisitem e aprofundem as ideias e conceitos iniciais formulados, sustentando um novo conhecimento a partir de múltiplos pontos de vista com teorização e discussão (COTTA RMM, et al., 2015).

O portfólio leva a mudanças em como as pessoas compreendem a si próprias e aos outros, valorizando a própria experiência, com a construção de uma identidade profissional. Estimula a reflexão sobre o cotidiano e a resolução criativa dos problemas vivenciados, subsidiando a tomada de decisões. Com o portfólio é possível a visualização da evolução, verificação de erros, análise de problemas e soluções alternativas, relacionando teoria e prática levando ao pensamento crítico. Permite o diálogo e a apreciação de ideias a partir do agir na prática (FORTE FDS, et al., 2015).

É uma avaliação mais concisa e fidedigna das competências adquiridas. O aluno torna-se autor da sua própria competência, assimilando conhecimento, desenvolvimento de habilidades, desempenho de atitudes de forma conjunta com seu avaliador. Determina uma avaliação contínua com papel central no alinhamento do ensino, aprendizagem e avaliação para aprimorar o conhecimento (LANA LD, 2015).

\section{Tipos de portfólios}

Não existe uma definição ou um padrão de portfólio ideal, a escolha depende dos objetivos a serem alcançados, onde o aluno documenta, registra e estrutura as ações por meio de um discurso crítico e reflexivo elaborado de forma contínua (COTTA, RMM, et al., 2016).

Existem inúmeras classificações e subtipos conhecidos de portfólios, como por exemplo o particular utilizado pelo professor, o de apresentação que ilustra as competências do aluno, resultados por ele alcançados, os de aprendizagem que contém as atividades e demonstram o percurso na apropriação do 
conhecimento, evidenciando dificuldades, tentativas de superação e a evolução. Existem ainda os portfólios demonstrativos que mostram importantes avanços, problemas que persistem, podendo revisitar os trabalhos construídos, auxiliando na compreensão e oferecendo subsídios para a construção de propostas de ensino. O portfólio de avaliação determina claramente os objetivos, define critérios para avaliação e decisões a serem tomadas, portfólio de trabalho reúne atividades guiadas pelos objetivos de aprendizagem em torno de um tema determinado para atingir objetivos curriculares (MAK P, et al., 2017)

Todos os portfólios compartilham três características básicas que permitem que eles sejam usados como uma ferramenta de avaliação: habilidade para coletar materiais criados ao longo do tempo, capacidade de selecionar desta coleção e organizá-la, e capacidade de envolver o trabalho com informações e conteúdo adicionais, para lembrar que portfólios são mais do que apenas arquivos gigantes (GOLDSMITH DJ, 2018).

\section{Finalidades dos portfólios}

Os objetivos do portfólio incluem avaliar e/ou acompanhar a aprendizagem, habilidades, competências, desenvolvimento profissional continuado, e estimular reflexão sobre as experiências. Serve para aproximar aluno e professor continuamente no processo de ação-reflexão-ação, com autoavaliação, espaço para dúvidas e conflitos, estímulo a narrativas e mobilizando o aluno para a responsabilidade sobre seu processo de aprendizagem, através da análise de peculiaridades do desenvolvimento (COTTA RMM, et al., 2016; SILVA CJV, et al., 2016).

O portfólio transforma o momento de avaliação em etapa de aprendizagem, pois avalia o progresso com diálogo, elaboração de novos conhecimentos e novas reflexões, criatividade, autonomia e empoderamento, com interação com professor. A avaliação torna-se um momento de troca. O aluno é autor da sua própria trajetória na construção do conhecimento, respeitando os limites e individualidades. E para a avaliação ser justa e fidedigna o docente precisa acompanhar efetiva e individualmente. Todos ensinam e aprendem mutuamente (CESARIO JB, et al., 2016), pode ser usado em diferentes cenários de aprendizagem. (FORTE M, et al., 2016) e o mais importante elemento da aprendizagem está na sua construção e não no seu produto (BERNARDI MC, et al., 2015). É também uma ferramenta de avaliação institucional, por isso a necessidade do apoio para a manutenção e ampliação do uso dessa ferramenta a partir da qualificação dos professores (PEREIRA EG, et al., 2015).

A autonomia é ponto-chave no processo de construção do portfólio, pois há liberdade de expressão, busca ativa e criatividade na elaboração de atividades, reflexão e crítica, para conseguir aprender além dos conteúdos, ampliando os horizontes. É fundamental que, para o sucesso do portfólio, haja clareza dos critérios de avaliação. O objetivo da avaliação é melhorar e não provar. Deve existir uma matriz para esclarecer os alunos e professores sobre os critérios de avaliação. Deve-se ter em mente o porquê da sua existência, a importância da reflexão no desenvolvimento profissional contínuo. Os alunos estabelecem metas de aprendizagem e monitoram, regulam e controlam o processo, construindo ativamente conhecimentos e habilidades (MAK P, et al., 2017).

O único cuidado é não tornar um projeto com apenas acúmulo de material, tem que haver finalidade específica, avaliar a qualidade do trabalho, identificar lacunas de conhecimento, permitir a autoavaliação e reflexão, alcançar progresso e competências (CLARKE JL, et al., 2016). O portfólio fornece um gatilho para provocar mudanças nas práticas de avaliação e exige uma grande mudança de pensamento (WANG $P$, et al., 2016), fornecendo as instituições uma avaliação autêntica da aprendizagem mais profunda com reflexão mais profunda (BAIRD K, et al., 2016).

\section{Vantagens e Desvantagens dos portfólios}

O portfólio tem inúmeras vantagens: é um potencializador de formação do pensamento compreensivo, crítico e criativo, melhora capacidade de alcançar objetivos e de integrar teoria e prática, melhora autoconsciência e capacidade de refletir, melhora capacidade de aprender de forma independente, tomada de decisão, pensamento crítico, aprimoramento de habilidades de comunicação, melhora autoconfiança, disposição para assumir responsabilidade sobre sua aprendizagem e profissionalismo. $O$ aluno satisfeito com 
seu processo de aprendizagem adquire mais conhecimento, aumenta a compreensão da teoria com aprendizagem mais aprofundada, tornam-se mais ativos com uma visão geral do seu progresso acadêmico (COTTA RMM, et al., 2015).

Para os professores desenvolve mais compreensão das necessidades dos alunos e muda a maneira de abordar o ensino, encoraja a reflexão, fomenta atitude crítica em relação ao próprio desempenho, aumenta a autoconfiança. O portfólio melhora também a relação aluno-professor, torna professores mais conscientes das necessidades, estimula o feedback e aumenta a confiança do aluno em compartilhar seus sentimentos em ambiente seguro, apoio emocional para situações difíceis (HEENEMAN S, et al., 2017).

Outras vantagens desta ferramenta são fornecer subsídios para o planejamento pedagógico, apontando desafios a serem superados, acompanhamento individual, oportunidade de se refazer nesse processo na construção da aprendizagem (FORTE FDS, et al., 2015). Permite a autoavaliação, com um processo de aprendizagem organizado e sequencial, compromisso com a superação através da reflexão continuada de forma enriquecedora, fugindo dos padrões tradicionais já esgotados e levando a momentos de maior interação entre alunos e professores (GENCEL IE, et al., 2016).

O portfólio possibilita, ainda, a tomada de consciência de forma continuada de seu processo avaliativo, desenvolvendo no estudante a responsabilidade e protagonismo de sua avaliação, articulando o saber pedagógico com saberes anteriormente adquiridos, além de proporcionar conhecimentos e reflexão (BAU C, 2019; GENCEL IE, et al., 2016), aumentando autoestima e confiança em um ambiente que oferece oportunidade de decisão com pensamento crítico e trabalho em equipe, identificando lacunas de aprendizado (TICHA V, 2015 e CHERTOFF J, et al., 2015).

Pode-se apontar como desvantagens a impossibilidade de execução devido rotina intensa e massificante, apreensão quanto ao modo de avaliar, objetivos não estarem claros e estabelecidos (STABENOW RSC, et al., 2015). Além da necessidade de capacitação do docente para o trabalho, requerendo muito tempo (COTTA RMM, et al., 2015) tem-se também a falta de conhecimento da real importância, complexidade, dificuldade em escrever e se expressar, dificuldade na reflexão das vivências, dificuldade em falar sobre si mesmos, escrever subjetividades, responsabilidade de entrega, provocando no professor inquietudes e no aluno certa desconfiança quanto ao resultado da avaliação, alguns preferindo o formato tradicional de avaliação (FORTE FDS, et al., 2015; CESARIO JB, et al., 2016; CHERTOFF J, et al., 2015). A falta de um modelo padronizado também seria uma dificuldade a implementação do portfólio (LANA LD, 2015).

\section{Reflexão e feedback}

O feedback é um momento privilegiado para ajuste no percurso formativo, pois ressalta aspectos positivos e negativos, estimula conhecimentos, habilidades e atitudes, discute narrativas, estimula o retorno a objetivos educacionais ainda não alcançados, levando ao desenvolvimento de senso ético e do cuidado integral em saúde (STELET BP, et al., 2016). Dentro do uso de portfólio é estratégico na percepção crítica da realidade destacando os pontos fortes do aluno, o que auxilia no aprimoramento da prática e oferecendo ambientes que permitam construir e reconstruir conhecimentos, desenvolver habilidades, competência profissional e mudanças nos processos de trabalho (LANA LD, 2015).

O feedback aponta os caminhos e estratégias para superação dos desafios e obstáculos, permitindo autoavaliação, avaliação interpares e avaliação docente a partir de critérios pactuados e coerentes com as competências que deseja desenvolver, gerando aprendizagem mais profunda, funcional, interessante e significativa (COTTA RMM, et al., 2016). Deve ter orientações quanto a pesquisas a serem feitas, contribuições construtivas. É uma oportunidade de corrigir a tempo (SILVA CJV, et al., 2016).

Pouco se estuda sobre como o feedback pode ser fornecido, recebido e internalizado para melhorar o aprendizado, facilitar o desenvolvimento e aumentar a motivação, para ter sucesso deve fornecer objetivos desafiadores e ajudar os alunos a alcançá-los, estabelecer metas, rever o progresso, planejar ações, monitorar o progresso, em direção ao objetivo facilitar o desenvolvimento da capacidade de autoavaliação para fechar lacunas de aprendizagem (LAM R, 2015). 
O feedback é o principal mecanismo de comunicação entre professor e aluno facilitando o auto monitoramento do seu trabalho (CLARKE JL, et al., 2016), com alunos auto direcionados e compreendendo melhor os objetivos de aprendizagem, critérios de avaliação, ajudando a planejar a aprendizagem futura e 0 domínio das habilidades (LAM R, 2015).

O portfólio também oferece uma oportunidade significativa para reflexão sobre o desenvolvimento contínuo, explorando as percepções do aluno, que refletem sobre pontos fracos e identificam o que pode melhorar. A prática reflexiva diminui a lacuna entre teoria e prática, atende as demandas de um contexto específico, inclui crescimento pessoal (TICHA V, 2015). A capacidade reflexiva é característica de um profissional competente, cria ponte entre teoria e prática, auxilia o pensamento crítico e a melhor compreensão para promover aprendizado autônomo e profundo através de questionamentos. Os profissionais precisam ser educados a refletir e o portfólio é excelente ferramenta para isso, pois exige que o aluno pense criticamente sobre as experiências, e a reflexão ajuda a esclarecer e dar sentido ao que foi aprendido, resolver incertezas, tomar decisões, aumenta autoconfiança, autoconsciência e habilidades de resolução de problemas, melhora comunicação e relação interpessoal (TONNI I, et al., 2016).

\section{CONSIDERAÇÕES FINAIS}

O portfólio promove um processo de reflexão crítica continuada, onde os estudantes constroem sua aprendizagem, relatam as vivências, dialogam com os problemas e superam as dificuldades. Não é apenas compilação, mas sim relevante processo das análises críticas das experiências vivenciadas. Estimula a autonomia, o espírito crítico, reflexivo e criativo, é um método didático reestruturador do processo de ensino e aprendizagem e avaliação por competências. É uma avaliação contínua e longitudinal levando a reflexão sobre os progressos alcançados, traçando novas metas e estratégias para o desenvolvimento das potencialidades. Favorece a criatividade num processo dinâmico e inovador potencializando a aprendizagem significativa através do feedback constante e da descrição da trajetória de aluno que é protagonista deste processo. Seu foco é o aprender em meio à diversidade e de forma contextualizada. É uma ferramenta útil para promover aprendizagem através da avaliação, incentiva aprendizagem ativa e promove a independência do aluno. Monitora qualidade, permite reflexão, avalia competências.

\section{REFERÊNCIAS}

1- BAIRD K, et al. Assessment of the quality and applicability of an e-portfolio capstone assessment item within a bachelor of midwifery program. Nurse Education in Practice, [s.I.], 2016; 20:11-16.

2- BAÚ AC. O portfólio como possibilidade de avaliação e reflexão. São Vicente do Sul, Disponível em: <https://www.portaleducacao.com.br/conteudo/artigos/biologia/o-portfolio-como-possibilidade-de-avaliacao-ereflexao/58063>, Acesso em: 25 jun, 2019.

3- BERNARDI MC, et al. Portfólio na avaliação do estudante de graduação na área da saúde: Estudo bibliométrico. Cogitare Enfermagem, Curitiba, 2015; 20(1): 153-160.

4- BOTTI SHO, REGO S. Processo ensino-aprendizagem na residência médica. Rev. bras. educ. med., Rio de Janeiro, 2010; 34(1): 132-140.

5- CASTRO-SILVA II, et al. Uso do portfólio na avaliação para aprendizagem na disciplina de Didática do Ensino Superior. In: CONGRESSO IBERO-AMERICANO EM INVESTIGAÇÃO QUALITATIVA, 7., 2018, Fortaleza. Atas. Fortaleza: Ludomedia, 2018; 1:1 - 9.

6- CARDOSO DAS, et al. Aprendizagem Reflexiva: o Uso do Portfólio Coletivo. Revista Brasileira de Educação Médica, [s.I.], 2015; 39:(3): 442-449.

7- CESÁRIO JB, et al. Portfólio reflexivo como estratégia de avaliação formativa. Revista Baiana de Enfermagem, [s.I.], 2016; 1(1): 356-364.

8- CHANG C, et al. Clinical nurse preceptors' perception of e-portfolio use for undergraduate students. Journal of Professional Nursing, [s.I.], 2017; 33(4): 276-281.

9- CHERTOFF J, et al. Status of portfolios in undergraduate medical education in the LCME accredited US medical school. Medical Teacher, [s.I.], 2015; 38(9): 886-896.

10- CLARKE JL, BOUD D. Refocusing portfolio assessment: Curating for feedback and portrayal. Innovations in Education and Teaching International, [s.I.], 2016; 55 (4): 479-486.

11- COTTA RMM, et al. Portfólio reflexivo: uma proposta de ensino e aprendizagem orientada por competências. Ciênc. saúde coletiva, Rio de Janeiro, 2013; 18(6): 1847-1856. 
12- COTTA RMM, et. Portfólios crítico-reflexivos: uma proposta pedagógica centrada nas competências cognitivas e metacognitivas. Interface - Comunicação, Saúde, Educação, [s.I.], 2015; 19(54): 573-588.

13- COTTA RMM, COSTA GD. Instrumento de avaliação e autoavaliação do portfólio reflexivo: uma construção teóricoconceitual. Interface - Comunicação, Saúde, Educação, [s.I.], 2016; 20(56): 171-183.

14- FORTE FDS, et al. Portfólio como estratégia de avaliação de estudantes de odontologia. Trabalho, Educação e Saúde, [s.I.], 2015, 13(2): 25-38.

15- FORTE M, et al. Portfólio Reflexivo Eletrônico: Resultados de um Projeto Piloto. Revista Brasileira de Educação Médica, [s.I.], 2016; 40(2): 234-244.

16- FRANK A, GIFFORD K. Electronic portfolio use in pediatric residency and perceived efficacy as a tool for teaching lifelong learning. Bmc Medical Education, [s.I.], 2017; 17(1): 1-5.

17- FROTA MMA, et al. O portfólio como estratégia facilitadora do processo de ensino-aprendizagem para a formação em odontologia. Adequação de metodologias de ensino utilizando o ambiente virtual de aprendizagem. Revista da Abeno, [s.I.], 2013; 11(1): 23-28.

18- GENCEL IE. The Effect of Portfolio Assessments on Metacognitive Skills and on Attitudes toward a Course. Educational Sciences: Theory \& Practice, [s.I.], 2016; 17(1): 293-319.

19- GOLDSMITH DJ. Enhancing learning and assessment through e-portfolios: A collaborative effort in Connecticut. New Directions for Student Services, [s.I.], 2007; 2007(119): 31-42.

20- HEENEMAN S, DRIESSEN EW. The use of a portfolio in postgraduate medical education - reflect, assess and account, one for each or all in one? Gms Journal for Medical Education, Maastricht, The Netherlands, 2017; 34(5): $1-12$.

21- LANA LD, BIRNER JA. Um relato de caso sobre a construção e elaboração do portfólio como metodologia avaliativa de aprendizagem. Ciencia y Enfermeria, Santa Maria, 2015; 21(3): 101-112.

22- LAM, R. Feedback About Self-Regulation: Does It Remain an "Unfinished Business" in Portfolio Assessment of Writing? Tesol Quarterly, [s.I.], 2015; 49(2): 402-413.

23- LAM R. Assessment as learning: examining a cycle of teaching, learning, and assessment of writing in the portfoliobased classroom. Studies in Higher Education, [s.I.], 2015; 41(11): 1900-1917.

24- MAK P, WONG KM. Self-regulation through portfolio assessment in writing classrooms. Elt Journal, [s.I.], 2017; 72(1): 49-61.

25- MCEWEN Laura A, et al. Developing and Successfully Implementing a Competency-Based Portfolio Assessment System in a Postgraduate Family Medicine Residency Program. Academic Medicine, [s.I.], 2015; 90(11): $1515-1526$.

26- O'BRIEN, CL, et al. Feasibility and Outcomes of Implementing a Portfolio Assessment System Alongside a Traditional Grading System. Academic Medicine, [s.I.], 2016; 91(11): 1554-1560.

27- PADOVANI O, CORREA AK. Tecnologias no uso do portfílio reflexivo em cursos superiores na área da saúde, In: CONVIBRA, 2015, IV Congresso Online - Gestão, Educação e Promoção da Saúde, 2015.

28- PEREIRA EG, et al. Portfólio no ensino em saúde: contribuição à reflexão a partir de seu uso na disciplina Enfermagem em Doenças Transmissíveis. Abcs Health Sciences, [s.I.], 2015; 40(3): 329-332.

29- COTTA RMM, et al. Portfólios coletivos como método de ensino, aprendizagem e avaliação crítico-reflexiva: exercitando o aprender a conviver e a trabalhar junto. In: CONGRESSO IBERO-AMERICANO EM INVESTIGAÇÃO QUALITATIVA, 5., 2016, Porto. Atas. Porto: Ludomedia, 2016;1: 17 - 26.

30- ROSSI NF, et al. As narrativas de estudantes de enfermagem nos portfólios do Estágio Curricular Supervisionado. Revista Eletrônica de Enfermagem, [s.I.], 2014; 16(3): 566-574.

31- SIDEBOTHAM M, et al. Preparing student midwives for professional practice: Evaluation of a student e-portfolio assessment item. Nurse Education in Practice, [s.I.], 2018; 32: 84-89.

32- SILVA CJV, et al. O portfólio como instrumento de autoavaliação crítico reflexiva na perspectiva dos alunos de um curso de medicina. Interdisciplinary Journal of Health Education, [s.I.], 2016; 1(1): 23-31.

33- STABENOW RSC, MAGALHÃES C. Portfólio como instrumento de avaliação formativa: a análise do processo no CMEI Valéria Veronesi. In: XVI Congresso da Educação e VI Simpósio de pesquisa e pós-graduação em educação, 2015, Londrina. Anais. Londrina, 2015; 638-649.

34- STELET BP, et al. Portfólio Reflexivo: subsídios filosóficos para uma práxis narrativa no ensino médico. Interface Comunicação, Saúde, Educação, [s.I.], 2016; 21(60): 165-176.

35- TICHA V, FAKUDE LP. Reflections on clinical practice whilst developing a portfolio of evidence: Perceptions of undergraduate nursing students in the Western Cape, South Africa. Curationis, [s.I.], 2015; 38(2): 1-8.

36- TONNI I, et al. Postgraduate Orthodontics Students' and Mentors' Perceptions of Portfolios and Discussion as Tools for Development of Reflection. Journal of Dental Education, Brescia, 2016; 80(9): 1098-1108.

37- VIEIRA VMO. Portfólio: uma proposta de avaliação como reconstrução do processo de aprendizagem. Psicol. Esc. Educ. (Impr.), Campinas, 2002; 6(2): 149-153.

38- WANG P, JEFFREY R. Listening to learners: An investigation into college students' attitudes towards the adoption of e-portfolios in English assessment and learning. British Journal of Educational Technology, [s.I.], 2016; 48(6): 1451-1463. 